Running Head: READING IN TIMES OF LOSS

\author{
Reading in Times of Loss: \\ An Exploration of the Functions of Literature during Grief \\ E. M. Koopman \\ Erasmus University Rotterdam
}

Author Note

E.M. Koopman, Department of Media and Communication, Erasmus University Rotterdam , The Netherlands.

E-mail: e.koopman@eshcc.eur.nl 


\title{
READING IN TIMES OF LOSS
}

\begin{abstract}
This study examined the use of literature as a coping strategy among people who had experienced loss, comparing it to the use of music. Theory suggested that literature and music might facilitate insight, recognition, support, relaxation, new emotions, and/or distraction. Among 198 respondents, 64 (32\%) reported that they used neither medium during their grief period, 65 (33\%) used only music, 19 (10\%) used only literature, and 50 (25\%) used both literature and music. Those who used both media reported a more impactful loss experience than the other groups. Respondents using neither medium scored lowest on preference for emotional coping. Most importantly, the pattern of group differences pointed toward a coping process that alternates between recognition and distraction, perhaps especially - but not only - by alternating between the distraction provided by reading and the recognition afforded by listening to music.
\end{abstract}

Keywords: loss, coping, grief, reading, literature, music, distraction 


\section{READING IN TIMES OF LOSS}

Reading in Times of Loss: An Exploration of the Functions of Literature during Grief

Grief constitutes an intense emotional experience that increases the risk of falling prey to illness or depression (Stroebe, Schut, \& Stroebe, 2007; Zisook \& Shuchter, 1991). Still, most people are able to cope with the loss of a beloved without professional help (Stroebe, Hansson, Stroebe, \& Schut, 2001). As a consequence, it is important to explore which “everyday" coping strategies - cognitive and behavioral efforts to alleviate stress (e.g. Folkman, Lazarus, Gruen, \& De Longis, 1986; Lazarus \& Folkman, 1984) - people use during grief. While seeking social support is an obvious and often effective coping strategy, it may not be readily available to everyone, and not everyone may feel like talking to other people when going through grief. An alternative coping strategy, which this article will examine, is the consumption of artistic media.

Within art therapy it has been stressed that creating an image or narrative gives people the opportunity to express feelings in a structured fashion (e.g. Eaton, Doherty, \& Widrick, 2007; Griffiths \& Corr, 2007). Yet, rather than creating images, melodies or stories oneself, most people will be more inclined to consume the art that others have made. Two particularly relevant forms of the consumption of artistic media are reading literature and listening to music. Literature is central to my current concerns, because I have studied it previously in the context of dealing with difficult life events (Koopman, 2011; Koopman, 2013) and because its benefits, while largely unexplored empirically, are continuously stressed by bibliotherapists and literary academics. For example, as Cohen (1992) has suggested in an anecdotal bibliotherapy study, the experience of recognition through reading can be similar to the experience of recognition derived from involvement in a support group. Recognizing one's own feelings could also play a role when listening to music, although music has more often been considered an effective way to regulate (rather than recognize) emotions (e.g., 


\section{READING IN TIMES OF LOSS}

Saarikallio \& Erkkilä, 2007). The current study attempts to establish the functions that reading fulfills after a loss, contrasting these functions with those that music listening fulfills.

\section{Theoretical background}

One of the main theories of how people deal with their loss experience, Stroebe and Schuts Dual Process Model of Coping with Bereavement (DPM; see Stroebe \& Schut, 1999; Stroebe \& Schut, 2001; Stroebe \& Schut, 2010), emphasizes the importance of both confrontation and distraction. Stroebe and Schut (2001) suggest that oscillating between confronting and being distracted from the loss (both the loss experience itself and its practical consequences) is the most effective way of coping. Distraction is theorized here as active defense. In fact, Stroebe and Schut tend to use the word "avoidance" and speak of the "confrontation-avoidance process" (e.g., Stroebe and Schut, 2001, p. 395). If either of the two dominates - one is either constantly ruminating about the loss or one is constantly trying to block it out - Stroebe and Schut (2001) expect a higher risk of pathological grief. In addition, they suggest that it is healthy to oscillate between positive and negative (re)appraisals of the loss experience and between focusing on the loss itself ("loss-oriented"; e.g., attending to loss-related emotions) and on its practical consequences ("restoration-oriented"; e.g., attending to loss-related life changes).

Since literature and (particularly) music appear of minor relevance to restorationoriented coping, the current study focuses specifically on the role of reading and listening to music in loss-oriented coping. When it comes to the loss experience itself, literature and music may either facilitate distraction from or confrontation with loss-related emotions. However, to what extent people use these media for either distraction or confrontation remains to be seen. 


\section{READING IN TIMES OF LOSS}

Two basic coping-preferences identified by Lazarus and Folkman (1984) are "emotion-focused coping" (attending to emotional effects of the loss) and "problem-focused coping ( solving practical problems caused by the loss) (see also Folkman, 1997, 2001). Problem-focused coping is "goal-directed", and "includes strategies for gathering information, decision making, planning, and resolving conflicts" (Folkman, 1997, p. 1213), while emotionfocused coping concentrates on the regulation of distressing emotions. While these basic coping preferences may seem to coincide with loss- and restoration-oriented coping, Stroebe and Schut (2010) have emphasized that they should not be conflated. Indeed, an individual engaged in restoration-oriented coping could be focused on emotion regulation, and an individual who is focused on the loss experience could be dealing with it as if solving a problem. In fact, it could be expected that people who are reading (fiction, not self-help books) or listening to music are more emotion-focused than problem-focused in their orientation toward the loss experience.

However, this expectation is largely based on common sense, since the supporting role of literature during grief is underexplored, as are the beneficial effects of literature on mental health generally. While there is some evidence from studies of bibliotherapy that reading literature can help people deal with a range of problems, from low self-esteem to adapting to a divorce (e.g., Schrank, 1981; Early, 1993; Kramer \& Smith, 1998), the mechanisms underlying these beneficial effects require further empirical clarification. In an initial exploration of the psychological processes involved in bibliotherapy, Shrodes (1950) argued on the basis of a number of case studies that readers arrive at insight and catharsis through a process of identification with characters in the narrative world. This resembles the kind of emotional grief work that is done during loss-oriented coping. However, this identification hypothesis has not been scrutinized more systematically (see Pehrsson \& McMillen, 2005). 


\section{READING IN TIMES OF LOSS}

Likewise, little is known about how readers in mental distress use literature outside of the therapeutic setting. Bernstein and Rudman (1989) reported how, in a non-clinical setting, reading literature can help children during grief. Based on results of other (anecdotal) bibliotherapy studies, these authors speculated that reading during grief can have the following emotional and cognitive functions, all of which are loss-oriented: (1) identifying with characters who are in a similar situation or have similar problems furthers understanding of or insight into one's own loss; (2) identification can also provide a sense of recognition and support by affirming that one is not alone in grief; (3) reading about new events and characters can provide temporary distraction from the complications of grief; (4) empathizing with characters helps to express feelings of sadness, anger and fear, possibly leading to a sense of relief (catharsis); (5) reflecting on how one's own situation can be compared to the narrative world can lead to further personal insight; and (6) the narrative can offer words that one did not yet have, making it easier to give words to grief and leading to better understanding / insight (Bernstein \& Rudman, 1989). Of these functions, only the third (temporary distraction) implies direct avoidance of the loss; all the others imply confrontation. All of the functions Bernstein and Rudman (1989) discuss in the context of children's grief could also be applicable to adults. In fact, empirical research by Zeelenberg and Spiertz (1993) into the reasons why adults with depression choose to read arrives at a similar list of reading's functions. Zeelenberg and Spiertz (1993) conducted a survey among a Dutch population of people suffering from depression, including one group who had tackled their depression without help from others. These respondents had chosen to read books themselves, either fiction, non-fiction or both. For them, recognition turned out to be the most important self-reported reason to read, and this rationale for reading coincided with a need for insight, advice and support (Zeelenberg \& Spiertz, 1993). These respondents thus sought confrontation, because of a need for both emotion-focused and problem-focused coping. The 


\section{READING IN TIMES OF LOSS}

severity of the depression mattered: those suffering from milder forms of depression reported more insight through reading than those suffering from grave depression.

Zeelenberg and Spiertz (1993) did their research on depression, not on grief. Even though the symptoms of a normal grief reaction correspond partially to those of depression, depression and grief are not identical (Zisook \& Kendler, 2007). Moreover, Zeelenberg and Spiertz (1993) were not specifically interested in literature: they also looked at self-help books and made no clear distinction between the different functions of different types of books. One relevant study that did focus on literature and grief was reported by Kuiken, Miall and Sikora (2004). Kuiken et al. (2004) presented participants with a poem by Coleridge, and found that for readers who had suffered a severe loss at least two years earlier, higher reported depression was associated with: (1) higher reports of "feeling resonance" (either literal comparison or metaphoric identification with feelings in the poem; p. 195) and (2) higher reports of personal insight ("self-perceptual depth"; p. 195). Yet, for those respondents dealing with a more recent significant loss (in the last year), higher depression was associated with lower resonance and lower insight. According to Kuiken et al. (2004), this lack of meaningful reading among the recently bereaved can be explained by the "numbness and derealization" in the initial period of the grieving process (p. 196). As time passes, a significant loss may become more accessible to reflection, possibly due to a decline in depression (cf. Zeelenberg \& Spiertz, 1993). In a later study, Sikora, Kuiken and Miall (2010) replicated the finding that the amount of time past after the loss matters: readers who had experienced loss two or more years before participation were more likely to attend to the stylistic features of the poem, leading them to metaphorically identify with characters in the text and subsequently report deepened self-perception.

While these studies provide deeper insight into how grief can influence the reading experience of poetry, they are just a first step when it comes to exploring reading as a coping 


\section{READING IN TIMES OF LOSS}

mechanism for those who are grieving. The experimental setting carries the disadvantage of decreased ecological validity: these respondents did not pick out a poem themselves but were presented with it. In contrast, a survey study by Koopman (2011) explored how people cope with difficult life events through reading, asking respondents to think back to a troublesome period in their lives in which a self-selected literary work had provided comfort or support. This study used questionnaire items based on the functions of reading during hardship mentioned by Bernstein and Rudman (1989) and Zeelenberg and Spiertz (1993). The explored functions were: distraction (from one's problems through reading about someone else's), recognition (of one's problems, feelings, and/or experiences in the work's characters and themes), insight (becoming more aware of the content of one's problems/feelings, experiencing increased self-understanding and becoming able to put one's feelings into perspective), and catharsis (becoming more at ease through reading, feeling relieved of negative emotions). In addition, the roles of "narrative emotions" and "aesthetic emotions" were explored. Narrative emotions are affective responses directed towards characters and the narrative world, like identification and empathy; aesthetic emotions are affective responses evoked by the style of the text (see Kneepkens \& Zwaan, 1994; Miall \& Kuiken, 2002; Tan, 1996).

Results of the survey study (Koopman, 2011) indicated that the most important predictor of "insight" (feeling awareness, self-understanding, broader perspective) was “recognition" (finding one's problems or feelings in story characters or story themes) and that, while narrative emotions correlated with insight, aesthetic emotions did not. The relation found between insight and recognition concurs with the expectations of theories of bibliotherapy (cf. Bernstein \& Rudman, 1989): if you can recognize your own feelings and problems in the characters or themes in a work of fiction, you are more likely to gain understanding of these feelings and problems. This also was also evident in a recent, more 


\section{READING IN TIMES OF LOSS}

qualitative survey study (Koopman, 2013) into readers' motives for reading an autobiographical novel about someone else's grief. Those who were also grieving primarily read to recognize their own experiences; by relating the author's experiences to their own, they found comfort as well as clarification of their own feelings and problems. This type of insight was compared to Aristotle's conception of "catharsis" as "clarification" rather than as "purgation" (e.g., Nussbaum, 1986). While the respondents who were grieving did not show a deeper understanding of life in general, they did show an increased understanding of their own experiences. Catharsis as clarification can be distinguished from the kind of distress alleviation (i.e., relaxation) provided by distraction or by focusing on someone or something other than themselves.

This distinction between the beneficial effects of distraction and the beneficial effects of confronting one's problems (through recognition), brings me to the current study. The present research explores these reactions (and others) among those who are grieving. However, instead of focusing on one particular novel, the present study looks at reading behavior more generally, exploring the functions of reading during grief and how diverse reading experiences are related to narrative and aesthetic emotions. Rather than hardships in general, the present study focuses on one kind of "hardship," namely, grief as a result of loss. Furthermore, it expands upon the previous study (Koopman, 2011) by not only exploring reading literature (novels, stories, and/or poems) but also listening to music as a loss-oriented coping strategy. Literature remains the main interest; music is mainly used for contrast, i.e., to identify those functions that may be unique for literature.

There is empirical evidence that music can help to regulate emotions, including mood improvement, distraction and relaxation, and better understanding of one's feelings and thoughts (e.g., Beck, 1991; Cassileth, Vickers, \& Magill, 2003; DeNora, 1999; North, Hargreaves, \& O’Neill, 2000; Wells \& Hakanen, 1991; Saarikallio, 2011; Saarikallio \& 


\section{READING IN TIMES OF LOSS}

Erkkilä, 2007). However, most of these studies have been restricted to adolescents and little attention has been given to the comparison between music and other media, like literature. Music with lyrics could be expected to fulfill similar functions to literature (recognition and insight), while instrumental music could be expected to change one's mood (evoking new emotions or calming one down).

Taking the preceding theoretical and empirical considerations into account, the following hypotheses were explored:

1. Both listening to music and reading are related to a need for emotion-focused coping, but not to a need for problem-focused coping.

As artistic media, both literature and music offer the opportunity for emotional engagement. They appear less relevant for problem-focused coping, since they do not offer direct practical advice. It remains to be seen whether the use of one of these media is more strongly related to emotion-focused coping.

2. Insight is a more important function for reading than for listening to music.

In the literature, insight is deemed to be an important function of reading (e.g. Bernstein \& Rudman, 1989; Shrodes, 1950; Zeelenberg \& Spiertz, 1993). While music has also been linked to reflection and understanding (e.g. DeNora, 1999; Saarikallio \& Erkkilä, 2007), it offers less elaborate narratives. Relatively, then, listening to music (perhaps especially instrumental music) offers less opportunity to learn from another person's problems.

3. A stronger experience of identification (specific to characters) and general recognition when reading is related to greater insight. Appreciating style will not be related to insight. 


\section{READING IN TIMES OF LOSS}

If one strongly identifies with characters, or generally recognizes oneself in the work, one may also be more likely to learn from what one reads (cf. Andringa, 2004; Bernstein \& Rudman, 1989; Koopman, 2011). A previous study (Koopman, 2011) showed a relation between recognition and insight - but not a relation between appreciating style and insight. A replication of these results was expected.

4. People are most likely to read when the impact of the grief is moderate.

Given the cognitive effort reading takes, and the impairment of cognitive functioning (i.e. numbing and problems with attention and focus) immediately after a significant loss (cf. Kuiken et al., 2004; Zeelenburg \& Spiertz, 1993), people may be less inclined to purposefully read when their loss is experienced as severe. On the other hand, when the loss they experienced (either recently or longer ago) is considered to be of less than moderate severity, there will be little need to read in order to work through the grief.

5. The impact of the loss will be negatively related to recognition and insight when reading if the loss is recent and severe: those who read shortly after a significant loss will experience less recognition and less insight than those who read after a loss with moderate impact.

As Kuiken et al. (2004) and Sikora et al. (2010) showed, having recently experienced a significant loss is related to feeling less resonance with the narrative world and experiencing less insight when reading literature (cf. Zeelenberg \& Spiertz, 1993).

\section{Methods}

\section{Participants and procedure}




\section{READING IN TIMES OF LOSS}

Initially, respondents were recruited through the Dutch public transport system, on six different trajectories. Train travelers above the age of 18 were approached by two researchers, the author of this article and a fellow graduate student. They were asked whether they were willing to participate in a study of reading and listening to music during grief. After signing an informed consent form, respondents filled out a questionnaire about the most significant loss they had experienced (either through death or divorce/separation), and whether they had purposefully used literature, music, both, or neither to work through their loss. This took them about 15 minutes, during which the researchers waited in a different wagon. Respondents did not receive any reimbursement for participating in the study. 125 train travelers handed in a questionnaire, of which 113 were valid. Questionnaires were judged to be invalid when respondents indicated they had not had a significant loss experience, or had failed to answer three or more questions (apart from those that were not applicable to their situation).

To increase the number of readers in the study, we also approached people who were likely to read; additional respondents were recruited through the public library of Utrecht and through calling or emailing book clubs that were found online. These respondents received the same information about the study as the train travelers and were free to stop participating in the study at any time. Of the people visiting the library, 46 handed in a questionnaire, of which 40 were valid. The book clubs added 52 questionnaires, of which 45 were valid.

Of this final group $(N=198), 64$ respondents indicated that they used neither medium (group 1), 65 people indicated that they only listened to music (group 2), 19 people indicated that they only read (group 3), and 50 people indicated that they both listened to music and read (group 4). Given the manner of recruiting, the final sample cannot be expected to be representative of the Dutch population. The total proportion of readers in this sample will be 


\section{READING IN TIMES OF LOSS}

overestimated compared to the total population. The total sample is varied, however: there is an almost equal number of males $(47.0 \%)$ and females $(53.0 \%), 44.9 \%$ of the respondents had received an education below vocational university level, and age ranged between 18 and 77 years $(M=39.3, S D=18.89)$. The demographic differences between the four groups can be found in Table 1. The difference in age was significant $\left(F(3,200)=6.77, p<.05, \eta^{2}=.09\right)$, with Post-hoc tests (Tukey HSD) showing that the "Music only" group had a lower average age than the "Literature only" group $(p<.05)$ and the "Both" group $(p<.001)$. Readers in this sample were older than music listeners, but they were not significantly older than the respondents who used neither medium. The gender-difference for the four groups bordered on significance: $\chi^{2}(3, N=204)=7.67, p=.053$. Since the "Literature only" group was relatively small, the analysis was repeated, merging the groups "Literature only" and "Both," thus creating three groups (Neither, Music only and All readers). For All readers, the genderdifference was significant, though the phi coefficient was not particularly large: $\chi^{2}(2, N=204)$ $=7.51, p=.023, p h i=.19$. Finally, there was a significant difference in educational level between the four groups $\left(\chi^{2}(3, N=204)=10.64, p=.014, p h i=.23\right)$; both groups who read were more highly educated than the other two groups. These demographic differences seem to reflect real-life differences, as previous sociological research has found readers to be more likely to be female, older, and higher educated (e.g., Cloïn et al., 2011; Zill \& Winglee, 1988).

Table 1: Age, gender, and education, for each of the four groups (neither, music, literature, both)

\begin{tabular}{lllll}
\hline & Neither & Music only & Literature only & Both \\
\hline$(N=198)$ & 64 & 65 & 19 & 50 \\
\hline$M(S D)$ age & $40.2(20.3)$ & $31.8(17.2)$ & $45.0(17.8)$ & $45.6(16.9)$ \\
Age (range) & $18-77$ & $18-74$ & $21-63$ & $18-72$ \\
\hline$N$ male & 36 & 34 & 5 & 18 \\
$N$ female & 28 & 31 & 14 & 32 \\
\hline
\end{tabular}




\section{READING IN TIMES OF LOSS}

\begin{tabular}{lllll}
\hline$N$ university & 34 & 27 & 12 & 36 \\
$N$ below university & 30 & 38 & 7 & 14 \\
\hline
\end{tabular}

\section{Measures}

Apart from the basic demographic questions, the questionnaire addressed the following topics: loss experience, coping style preference, and questions regarding reading literature (defined as "novels, stories or poems") and listening to music (of any genre).

\section{Questions regarding loss experience}

Respondents were asked to report on one loss experience, the one "that has influenced you the most during recent years." Items addressed the manner of separation (either death or partner divorce/break-up) and how long ago the loss had taken place ("recency"). Questions on the impact of the loss were based on items from the Dutch version of the Inventory of Traumatic Grief (ITG) (Boelen, Van den Bout, De Keijser, \& Hoijtink, 2003). Impact of the loss was measured through the three following questions (7-point scale), which were also used to measure impact within the ITG: "To what extent has this loss influenced your life?", "How hard has this loss been on you?", and "To what extent did you find it difficult to deal with the loss?" In this study, the three items had a Cronbach's alpha of 0.88 , allowing them to be combined into one construct: "impact".

Questions regarding coping style

To measure whether people's coping style was more problem-focused or more emotionfocused, two 7-point rating scales were used: “Are you, generally, someone who likes to find practical solutions when you have sorrows or problems?" (problem-focused coping), and "Are you, generally, someone who finds it important to take the time to consider the emotional consequences of problems?" (emotion-focused coping). While these items were based on the distinction made between problem-focused and emotion-focused coping by Lazarus and Folkman (e.g. Lazarus \& Folkman, 1984), I did not use their Ways of Coping 


\section{READING IN TIMES OF LOSS}

Questionnaire (Lazarus \& Folkman, 1985), since this questionnaire contains 57 items and would thus be too time-consuming to answer for the respondents in this survey. For the purposes of this study (i.e., an exploration of the role of coping style when reading during grief), using these two more generalized formulations to capture the gist of the two constructs was considered sufficient.

\section{Questions regarding music and literature}

The main part of the questionnaire consisted of questions about music and reading. For music, the following yes/no-question was asked first: "In order to deal with your loss, have you purposefully used music?” The same question was asked for literature, replacing "music" with "a novel, story and/or poem." Those who answered such a question in the negative were referred to the next section. In this way, most people were expected to be able to fill out the questionnaire, allowing for comparisons between those who have and those who have not used literature. After the selection-question, questions followed on the different potential functions, all on a 7-point scale. Items were based on the functions proposed by Bernstein and Rudman (1989), the self-reported functions by depressed readers in the Zeelenberg and Spiertz study (1993) and the items used in the Koopman (2011) study. In addition to this, research on the emotion-regulating function of music (Saarikallio \& Erkkilä, 2007) gave rise to an item on "new emotions", which was also deemed potentially relevant for literature (cf. Cupchik, Oatley, \& Vorderer, 1998):

1. Distraction: "Music/ reading offered me distraction from my sorrow, anxieties and/or grief."

2. Insight, consisting of four items:

- "Listening to music/ reading made me aware of feelings and/or thoughts I previously did not understand.” (Awareness) 


\section{READING IN TIMES OF LOSS}

- "Listening to music/ reading made me better able to accept the loss." (Accepting the loss)

- "Listening to music/ reading helped me to give words to my own feelings." (Giving words)

- "Listening to music/ reading helped me to put the loss in perspective." (Put in perspective)

Both in the case of literature and of music these four items could be taken together to form one construct: $\alpha=.98$ for literature, and $\alpha=.96$ for music.

3. Support: "Listening to music/ reading gave me the feeling I was not alone in my sorrow."

4. Recognition: "The music/ reading offered me a sense of recognition."

5. New emotions: "Listening to music/ reading made me experience new emotions."

\section{Relaxation: "After reading I felt calmer.,"}

To explore which aspects of the work influenced the above-mentioned functions in the case of literature, particularly to see whether style and/or narrative feelings are of significance, respondents were asked about their subjective experience of the text they had read. On a 7point scale they could indicate to what extent they had found style important ("I could appreciate the beauty of the language"), and to what extent they had experienced the narrative emotions "identification" ("I recognized myself or my own situation in the characters") and "empathy" ("I empathized with the characters and/or narrator"). For music, the main question relevant to the study of literature was to what extent respondents had found the lyrics important.

\footnotetext{
${ }^{1}$ This last question was only included in the items on literature, not in those on music. This was an error in the design, as a consequence of the fact that two researchers conducted two separate studies with the same questionnaire.
} 


\section{READING IN TIMES OF LOSS}

\section{Results}

\section{Coping preference}

The first hypothesis stated that both listening to music and reading would be related to a need for emotion-focused coping, but not to a need for problem-focused coping. One-way ANOVA's confirmed this hypothesis. While the four groups did not differ on their preference for problem-focused coping $\left(F(3,194)=1.51, p=.21, \eta^{2}=.02\right)$, there was a significant group difference for emotion-focused coping: $F(3,194)=10.35, p<.001, \eta^{2}=0.14$. Post-hoc tests (Tukey HSD) showed that the mean score for the Neither group on emotional coping $(M=$ 3.83, $S D=1.43)$ was significantly lower than for the Music only group $(M=4.69, S D=1.63)$, the Literature only group $(M=5.00, S D=1.25)$, and the Both group $(M=5.28, S D=1.26)$. Thus, consuming artistic media during grief was related to a preference for paying attention to "the emotional consequences of problems."

Since there were more women in the higher scoring Literature only group and the Both group, and an emotional coping preference could be more prevalent among women, this may have influenced the aforementioned results. To test this, a two-way ANOVA was conducted for gender and group. Taking gender into account, there still was a significant difference between the four groups, with the same pattern of means, namely the Neither group scoring lower than all the other groups $\left(F(3,198)=8.37, p<.001, \eta^{2}=.12\right)$. Gender also had a main effect on emotional coping, with women scoring higher: $F(1,198)=8.06, p<.01, \eta^{2}$ $=.04$, but there was no significant interaction between gender and group $(F(3,198)=1.40, p$ $\left.=.24, \eta^{2}=.02\right)$.

\section{Functions of reading and music during grief}

Before looking at the directional hypotheses about the differences in functions between reading and listening to music, it is relevant to first examine which functions readers rated as particularly important. Friedman and Wilcoxon signed ranks tests were used to explore which 


\section{READING IN TIMES OF LOSS}

functions (distraction, insight, recognition, support, new emotions, and relaxation) scored highest among readers for the combined Literature only and Both groups.

The Friedman test was significant $\left(\chi^{2}(8, N=69)=68.49, p<.001\right)$, and provided the following rank order (means are given to indicate how respondents scored on a 7-point scale):

1. Distraction (Mean Rank: 6.12; $M=4.97, S D=1.63$ ),

2. Relaxation (Mean Rank: 6.02; $M=5.03, S D=1.19$ ),

3. Recognition (Mean Rank: 5.99; $M=4.77, S D=1.53$ ),

4. Awareness (Mean Rank: 5.04; $M=4.28, S D=1.80$ ),

5. Support (Mean Rank: 4.83; $M=4.14, S D=1.80$ ),

6. Accepting the loss (Mean Rank: 4.71; $M=4.12, S D=1.66$ ),

7. Put in perspective (Mean Rank: $4.39 ; M=3.97, S D=1.62$ ),

8. New emotions (Mean Rank: 4.01; $M=3.78, S D=1.62$ ),

9. Giving words (Mean Rank: 3.89; $M=3.74, S D=1.68$ ).

Wilcoxon signed ranks tests showed that readers rated the three highest ranking items significantly higher than the other six. "Distraction," "relaxation," and "recognition" are thus perceived by respondents to be the most important functions when reading during grief.

For those respondents who only listened to music, the Friedman test - which was also significant $\left(\chi^{2}(7, N=65)=34.659, p<.001\right)-$ provided the following rank order (note that "relaxation" was not included, since it did not figure as an item for music):

1. Recognition (Mean Rank: 5.73; $M=5.20, S D=1.51$ ),

2. Distraction (Mean Rank: $4.97 ; M=4.46, S D=1.63$ ),

3. Put in perspective (Mean Rank: $4.47 ; M=4.48, S D=1.50$ ),

4. New emotions (Mean Rank: 4.46; $M=4.37, S D=1.64$ ),

5. Support (Mean Rank: 4.45; $M=4.28, S D=1.68$ ),

6. Awareness (Mean Rank: 4.06; $M=4.17, S D=1.55$ ), 


\section{READING IN TIMES OF LOSS}

7. Accepting the loss (Mean Rank: 4.01; $M=4.09, S D=1.61$ ),

8. Giving words (Mean Rank: 3.85; $M=4.06, S D=1.65$ )

Wilcoxon signed ranks tests showed that music listeners rated "recognition" significantly higher than the seven other functions. Other significant differences were not found.

Together these results indicate that both readers and music listeners find recognition important, but for music listeners recognition stood out distinctively, while for readers both distraction and relaxation also stood out.

Since the total group of readers consisted of respondents approached in three different ways (train travelers, library visitors and book club members) and this background might influence the importance of different functions, a series of ANOVA's was conducted to check for differences between these three groups of readers. No significant differences were found.

\section{Different functions of literature and music}

The second hypothesis was that insight would be a more important function (score higher) for reading than for listening to music. Since the group sizes were unequal, there was an increased risk of type I errors. For the direct comparison of the functions of literature and music, I therefore chose to look at the "Both" group. Thus, to judge the relative importance of insight as a function of reading during grief, a comparison was made between reading and listening to music within the group that did both $(N=50)$. A Wilcoxon test was used to compare each pair of the functions ("relaxation" could not be compared, since it did not figure as an item for music). The results for the different functions can be found in Table 2. In line with the previously found rank orders, which did not suggest an important role for the insightitems for either readers or music listeners, there was no significant difference between literature and music for "insight" $(p=.75)$. Looking at the separate items of insight did not matter. However, there were significant differences for other functions: "distraction" scored 


\section{READING IN TIMES OF LOSS}

higher for reading $(p<.05)$, while "new emotions" $(p<.05)$ and "recognition" $(p<.01)$ scored higher for music.

Table 2: Different functions for literature and music

\begin{tabular}{lccl}
\hline$(N=50)$ & Literature & Music & $Z$ \\
& $M(S D)$ & $M(S D)$ & \\
\hline Insight (sumscore) & $15.60(5.90)$ & $15.92(5.62)$ & -.18 \\
Distraction & $4.97(1.64)$ & $4.46(1.54)$ & $1.95^{*}$ \\
New Emotions & $3.86(1.50)$ & $4.32(1.73)$ & $-2.09^{*}$ \\
Recognition & $4.68(1.48)$ & $5.40(1.18)$ & $-3.10^{* *}$ \\
Support & $3.96(1.64)$ & $4.28(1.63)$ & -1.41 \\
& & & \\
\hline
\end{tabular}

Note: $* p<.05, * * p<.01$

A possible explanation for the lack of difference for "insight" between literature and music could be respondents' attention to lyrics. Within the "Both" group, 27 respondents found lyrics important and 23 did not. Mann-Whitney tests showed that the respondents who found lyrics important did have higher scores on two insight-items for music, namely "awareness" and "giving words." These differences, however, only bordered on significance (respectively: $Z=-1.52, p=.12 ; Z=-1.67, p=.09$ ). While these results are suggestive, it could not be confirmed that reading leads to higher insight than listening to instrumental music.

\section{Correlations with insight}

The third hypothesis stated that for readers, higher scores on "recognition" and “identification" would coincide with higher scores on "insight". As can be seen in Table 3, readers (the Literature only and Both groups combined) indeed showed a significant correlation between recognition and the sumscore of insight, and between identification and insight. The third hypothesis also stated that appreciating the style would not be related to insight. Table 3 shows the Spearman correlations for all the main functions of reading as well 


\section{READING IN TIMES OF LOSS}

as stylistic appreciation and the narrative emotions identification and empathy. "Style" indeed did not correlate significantly with the sumscore of insight; neither did it correlate with the other functions, apart from distraction, with which it correlated moderately. Identification and empathy appeared more crucial; especially identification, which correlated with all functions except distraction.

Table 3: Spearman Correlations of the functions of reading, narrative emotions, and style

\begin{tabular}{|c|c|c|c|c|c|c|c|c|c|}
\hline$(N=69)$ & Insight & $\begin{array}{l}\text { Recogni- } \\
\text { tion }\end{array}$ & $\begin{array}{l}\text { Distrac } \\
\text {-tion }\end{array}$ & Support & $\begin{array}{l}\text { New } \\
\text { emotions }\end{array}$ & $\begin{array}{l}\text { Relaxa } \\
\text {-tion }\end{array}$ & Style & $\begin{array}{l}\text { Identi- } \\
\text { fication }\end{array}$ & Empathy \\
\hline Insight & & $.49 * *$ & -.14 & $.79 * *$ & $.52 * *$ & .19 & .18 & $.37 * *$ & .21 \\
\hline $\begin{array}{l}\text { Recog- } \\
\text { nition }\end{array}$ & & & -.06 & $.60 * *$ & .20 & $.24 *$ & .09 & $.64 * *$ & $.43 * *$ \\
\hline $\begin{array}{l}\text { Distrac- } \\
\text { tion }\end{array}$ & & & & -.15 & -.11 & $.28 *$ & $.34 * *$ & .07 & .15 \\
\hline Support & & & & & $.43 * *$ & .21 & .11 & $.47 * *$ & $.25 *$ \\
\hline $\begin{array}{l}\text { New } \\
\text { Emotions }\end{array}$ & & & & & & .22 & .08 & $.30 *$ & .11 \\
\hline $\begin{array}{l}\text { Relaxa- } \\
\text { tion }\end{array}$ & & & & & & & .16 & $.31 * *$ & $.42 * *$ \\
\hline Style & & & & & & & & .22 & $.31 * *$ \\
\hline $\begin{array}{l}\text { Identifi- } \\
\text { cation }\end{array}$ & & & & & & & & & $.45^{* * *}$ \\
\hline
\end{tabular}

\section{Coping with losses of different intensities}

The fourth hypothesis stated that people are most likely to read when the impact of the grief is moderate. To test this hypothesis, the sumscore of "impact" (range: 3-21) was cut-off to form three groups (low: 3-10, moderate: 11-15, high: 16-21). A Chi-square test was used to see how readers and non-readers were distributed over these three impact groups. As Table 4 shows, most of the readers were in the "high impact" group (49.3\%).

To get a more nuanced picture of the relationship between impact of the loss and use of artistic media, a one-way between-groups analysis of covariance (ANCOVA) was executed, measuring the sumscore of impact for all four groups, controlling for time passed since the loss had taken place (or: "recency"). 


\section{READING IN TIMES OF LOSS}

The ANCOVA confirmed a significant difference between the groups on reported impact: $F(3,195)=11.51, p<.001, \eta^{2}=.15$. The means were as follows: Neither: $M=10.77$; Music only: $M=12.41$; Literature only: $M=11.86$; Both: $M=15.17$. Post-hoc tests (Tukey HSD) showed that respondents in the group "Both" experienced a higher impact of the loss than all other three groups. How long ago the loss had taken place played no significant role: $F(1,195)=.57, p=.45, \eta^{2}=.003$.

In addition, there was a significant difference on impact of the loss between those who had lost a partner through divorce $(n=35)$ and those whose beloved was deceased $(n=163)$, with the divorcees reporting higher impact: $F(1,195)=12.50, p<.001, \eta^{2}=.06$. Controlling for this variable still resulted in a significant effect of artistic media use on impact (with "Both" scoring higher than the other groups), albeit with a smaller effect size: $F(3,198)=$ $5.46, p<.001, \eta^{2}=.08$.

Table 4: Distribution of readers and non-readers over three impact groups

\begin{tabular}{lccc}
\hline$(N=198)$ & \multicolumn{3}{c}{ Impact } \\
& Low & Moderate & High \\
\hline $\begin{array}{l}\text { Non-readers } \\
(n=129)\end{array}$ & 55 & 41 & 33 \\
& $(42.6 \%)$ & $(31.8 \%)$ & $(25.6 \%)$ \\
\hline $\begin{array}{l}\text { Readers } \\
(n=69)\end{array}$ & 12 & 23 & 34 \\
\hline$\chi^{2}(\mathrm{df})$ & $(17.4 \%)$ & $(33.3 \%)$ & $(49.3 \%)$ \\
\hline$P$ & \multicolumn{3}{c}{$15.96(2)$} \\
\hline
\end{tabular}

\section{Loss intensity, recognition, and insight}

The fifth and final hypothesis was that, for readers who had experienced a recent loss, a higher impact of the loss would be related to lower scores on recognition and insight. This hypothesis was not confirmed. Interestingly, the opposite pattern emerged: reading after recent and severe loss experiences appeared connected to recognition and insight. This result 


\section{READING IN TIMES OF LOSS}

was arrived at through an analysis of the entire sub-sample of readers, followed by an analysis of a smaller sub-sample of recent loss participants. Self-reported impact for all readers $(N=$ 69) did not show significant (Spearman Rank Order) correlations with insight $(r s=-.03, p=$ .79 ) and recognition $(r s=.18, p=.14)$. However, repeating the correlations for impact, taking into account only those readers who had suffered a relatively recent loss (in the last year: $n=$ 13), did lead to strong positive correlations, bordering on significance for impact and insight $(r s=.53, p=.06)$, and significant for impact and recognition $(r s=.56, p<.05)$.

\section{Discussion}

This study explored the potential of literature as a loss-oriented coping strategy during grief, contrasting it with listening to music. The general aims were to investigate the role of the various functions mentioned in bibliotherapy research (a.o. Bernstein \& Rudman, 1989; Zeelenberg \& Spiertz, 1993), to look into the relation between artistic media use during grief and coping needs, and to investigate the role of loss severity.

The current study indicates that using artistic media (literature, music or both) during the grief process is related to a particularly emotional experience of the loss in two ways. First, a preference for emotion-focused coping was greater among those who used either artistic medium during the grief process than among those who used neither medium. Those who used literature and/or music during grief had a higher need to pay attention to the emotional consequences of problems than those who used neither medium. There was no clear evidence that either literature or music is more important for those with a preference for emotion-focused coping.

Second, the impact of the loss was greater for the groups that had used artistic media, and significantly so for the group that had used both literature and music. Following cognitive stress theory (Lazarus \& Folkman, 1984), a more stressful experience may also require more, 


\section{READING IN TIMES OF LOSS}

and more varied, coping efforts. However, perhaps these respondents had a general tendency to use more artistic media and this predisposition was associated with greater emotionality, leading them to report both a higher emotional coping preference and higher loss impact. Possibly, the personality trait Openness, which was not measured in this study, could explain the relation between using artistic media and emotionality during grief. This trait measures both attention to one's own feelings and aesthetic sensitivity and has been related in previous research to the use of artistic media (e.g., McManus \& Furnham, 2006).

It was expected that high loss impact would impair cognitive abilities and consequently lead fewer people to read. On the contrary, however, respondents appeared more likely to read when the impact of the loss was high. In addition, the impact of the loss was related to experiencing more insight and more recognition through reading for those who had experienced a recent loss (in the last year). These findings seem directly opposed to those of Kuiken et al. (2004), Sikora et al. (2010), and Zeelenberg and Spiertz (1993).

The contrast with the Zeelenberg and Spiertz (1993) study may not be a contrast at all, since Zeelenberg and Spiertz looked into depression instead of grief. The group that suffered from severe depression in their study reported less insight than those suffering from milder depression. In the current study, those with the highest reported impact were unlikely to qualify as severely depressed, given the manner of approach (everyday settings: train, library, book clubs - places those who are gravely depressed may avoid). The contrast between the experimental studies and the current survey study may be mainly one of self-selection: participants in the Kuiken et al. (2004) and Sikora et al. (2010) studies were assigned to read a poem, while respondents in this survey indicated they had chosen to read during their grief process. If one is capable and willing to read, insight may not be impaired. The stronger need one may feel to read when the impact is higher could then even lead to higher insight. 


\section{READING IN TIMES OF LOSS}

However, the group that had read and had lost someone in the last year was rather small $(n=$ 13); so these results should be treated with caution.

The present study indicates that, as Shear (2010) has emphasized, distraction fulfills an important function during grief: it satisfies a need to escape from the stress and pain that loss causes. The importance of distraction in establishing a sense of relaxation in the context of reading has previously been shown in a study on reading during hardship (Koopman, 2011), and that function of distraction among readers has been confirmed in the present study. Specifically, among readers (including those who only read and those who also listened to music), distraction was among the most higly ranked coping functions - even though those who only listened to music also reported that distraction was important. It is noteworthy that, among those who both read and listened to music distraction was rated as a more important function for reading than it was for listening to music. Perhaps the cognitive effort required for reading facilitates this function: the words on paper only come to life with an effortful shift in attention from the loss experience to the narrative world.

Both media also support recognition. Among readers (including those who only read and those who also listened to music) and among those who listened to music, recognition was among the most highly ranked coping functions. Moreover, among respondents using both media, music was more likely than reading to provide recognition and new emotions. Taken together, these results suggest a coping process that alternates between recognition and distraction, perhaps especially - but not only - by alternating between the distraction provided by reading and the recognition afforded by listening to music. This is in line with the image of normal coping during grief as portrayed in the Dual Process Model of Coping with Bereavement (Stroebe \& Schut, 1999; Stroebe \& Schut, 2001; Stroebe \& Schut, 2010): seeking both confrontation with and relief from thoughts of the loss. The precise pattern of 


\section{READING IN TIMES OF LOSS}

alternation between these functions (for both media) requires a research design that follows respondents throughout the grief process.

Another function deemed important in the literature (e.g. Bernstein \& Rudman, 1989; Zeelenberg \& Spiertz, 1993), insight, is reportedly not very prevalent during the grief process. In addition, and against expectation, respondents who both listened to music and read literature were not more likely to report insight as a consequence of reading than as a consequence of listening to music. (This pattern was evident regardless of the impact of the loss.) One possible explanation for this pattern may be the lyrics of many musical genres. Those who found lyrics important scored higher on becoming aware of one's feelings and giving words to one's sorrow. While these differences were not significant, but bordering on significance, this does suggest that musical lyrics play a role in gaining insight.

Another possible explanation for why insight did not score higher for reading is the importance of the "recognition" function for music. The literature on bibliotherapy (e.g., Bernstein \& Rudman, 1989; Shrodes, 1950) assumes that it is through recognizing oneself in characters and themes that insight is attained. Empirical support for this assumption comes from Kuiken et al. (2004), from Koopman's earlier research $(2011 ; 2013)$ and again from the present study: "recognition" of oneself in the characters and themes of a narrative is related to insight. Perhaps this generalization should be extended to musical lyrics as well. Under what

circumstances (e.g., for which types of poems, novels or lyrics) such insight emerges, as well as the likely qualitative differences between insights gained from lyrics and from literature, are intriguing issues for future research.

This study, though correlational in nature, has given an important indication that both literature and music may help those who feel a need to attend to the emotional consequences of grief. To benefit clinical practice, further research should look into differences in specific coping strategies between people who are and who are not able to handle the experience of 


\section{READING IN TIMES OF LOSS}

loss. Given the ease with which any of us can pick up a book in combination with the many potential beneficial functions ascribed to literature, reading is a coping strategy that this type of research cannot afford to ignore.

\section{Acknowledgements}

This article is based on the research I did for my graduate thesis in Clinical Psychology at Utrecht University, under supervision of Henk Schut. Another graduate student, Elly van Dijk, cooperated on this project, looking into the functions of music. I would like to thank both of them for the fruitful collaboration.

\section{References}

Andringa, E. (2004). The interface between fiction and life: Patterns of identification in reading autobiographies. Poetics Today, 25, 205-240.

Beck, S. L. C. (1991). The therapeutic use of music for cancer-related pain. Oncology Nursing Forum, 18, 1327-1337.

Bernstein, J. E., \& Rudman, M. K. (1989). Books to help children cope with separation and loss. New York: R.R. Bowker.

Boelen, P. A., Van den Bout, J., De Keijser, J. \& Hoijtink, H. (2003). Reliability and validity of the Dutch version of the Inventory of Traumatic Grief (ITG). Death Studies, 27, 227-247.

Cassileth, B. R, Vickers, A. J, \& Magill, L. A. (2003). Music therapy for mood disturbance during hospitalization for autologous stem cell transplantation: A randomized controlled trial. Cancer, 98, 2723-2729.

Cloïn, M., Kamphuis, C., Schols, M., Tiessen-Raaphorst, A., \& Verbeek, D. (2011). 


\section{READING IN TIMES OF LOSS}

Nederland in een dag. Tijdbesteding in Nederland vergeleken met die in 15 andere Europese landen. Retrieved from http://www.scp.nl/Publicaties/Alle_publicaties/Publicaties_2011/Nederland_in_een_d ag

Cohen, L. J. (1992). Bibliotherapy: The therapeutic use of books for women. Journal of Nurse-Midwifery, 37, 91-95.

Cupchik, G. C., Oatley, K., \& Vorderer, P. (1998). Emotional effects of reading excerpts from short stories by James Joyce. Poetics, 25, 363-377.

DeNora, T. (1999). Music as a technology of the self. Poetics, 26, 1-26.

Early, B. P. (1993). The healing magic of myth: Allegorical tales and the treatment of children of divorce. Child and Adolescent Social Work Journal, 10, 97-106.

Eaton, L.G., Doherty, K.L., \& Widrick, R.M. (2007). A review of research and methods used to establish art therapy as an effective treatment method for traumatized children. The Arts in Psychotherapy, 34, 256-262.

Folkman, S. (1997). Positive psychological states and coping with severe stress. Social Science and Medicine, 45, 1207-1221.

Folkman, S. (2001). Revised coping theory and the process of bereavement. In Stroebe, M. S., Hansson, R. O., Stroebe, W., \& Schut, H. (eds.), Handbook of bereavement research: Consequences, coping and care (pp. 563-584). Washington, DC: American Psychological Association.

Folkman, S., Lazarus, R. S., Gruen, R. J., \& De Longis, A. (1986). Appraisal, coping, health status and psychological symptoms. Journal of Personality and Social Psychology, 50, $571-579$.

Griffiths, S., \& Corr, S. (2007). The use of creative activities with people with mental health problems: A survey of occupational therapists. The British Journal of Occupational 


\section{READING IN TIMES OF LOSS}

Therapy, 70, 107-114.

Koopman, E. M. (2011). Predictors of insight and catharsis among readers who use literature as a coping strategy. Scientific Study of Literature, 1(2), 241-259.

Koopman, E. M. (2013). The attraction of tragic narrative: Catharis and other motives. Scientific Study of Literature, 3(2), [pages unknown]

Kneepkens, E. W. E. M., \& Zwaan, R. A. (1994). Emotions and text comprehension. Poetics, $23,125-138$.

Kramer, P. A., \& Smith, G. G. (1998). Easing the pain of divorce through children's literature. Early Childhood Education Journal, 26, 89-94.

Kuiken, D., Miall, D. S., \& Sikora, S. (2004). Forms of self-implication in literary reading. Poetics Today, 25, 171-203.

Lazarus, R., \& Folkman, S. (1984). Stress, appraisal and coping. New York: Springer.

Lazarus, R., \& Folkman, S. (1985). If it changes it must be a process: Study of emotion and coping during three stages of a college examination. Journal of Personality and Social Psychology, 48, 150-170.

McManus, I.C., \& Furnham, A. (2006). Aesthetic activities and aesthetic attitudes: Influences of education, background and personality on interest and involvement in the arts. British Journal of Psychology, 97, 555-587.

Miall, D.S., \& Kuiken, D. (2002). A feeling for fiction: Becoming what we behold. Poetics, $30,221-41$.

North, A. C., Hargreaves, D. J., \& O’Neill, S. A. (2000). The importance of music to adolescents. British Journal of Education Psychology, 70, 255-272.

Pehrsson, D. E., \& McMillen, P. (2005). A bibliotherapy evaluation tool: Grounding counselors in the therapeutic use of literature. The Arts in Psychotherapy, 32, 47-59.

Saarikallio, S. (2011). Music as emotional self-regulation throughout adulthood. Psychology 


\section{READING IN TIMES OF LOSS}

of Music, 39, 307-327.

Saarikallio, S., \& Erkkilä, J. (2007). The role of music in adolescents' mood regulation. Psychology of Music, 35, 88-109.

Schrank, F. A. (1981). Bibliotherapy as a counseling adjunct. Personnel and Guidance Journal, 60, 143-147.

Shear, M. K. (2010). Exploring the role of experiential avoidance from the Perspective of Attachment Theory and the Dual Process Model. Omega, 61, 359-371.

Shrodes, C. (1950). Bibliotherapy: A theoretical and clinical-experimental study. Doctoral dissertation. University of California, Berkeley (Dissertation Abstracts Online).

Sikora, S., Kuiken, D., \& Miall, D. S. (2010). An uncommon resonance: The influence of loss on expressive reading. Empirical Studies of the Arts, 28, 135-153.

Stroebe, M. S., Hansson, R. O., Stroebe, W., \& Schut, H. (2001). Introduction: Concepts and issues in contemporary research on bereavement. Handbook of bereavement research: Consequences, coping and care (pp. 3-22). Washington, DC: American Psychological Association.

Stroebe, M. S., \& Schut, H. (1999). The Dual Process Model of Coping with Bereavement: Rationale and description. Death Studies, 23, 197-224.

Stroebe, M. S., \& Schut, H. (2001). Models of coping with bereavement: A review. In Stroebe, M. S., Hansson, R. O., Stroebe, W., \& Schut, H. (eds.), Handbook of bereavement research: Consequences, coping and care (pp. 375-403). Washington, DC: American Psychological Association.

Stroebe, M. S., \& Schut, H. (2010). The Dual Process Model of Coping with Bereavement: A decade on. Omega, 61, 273-289.

Stroebe, M. S., Schut, H., \& Stroebe, W. (2007). Health outcomes of bereavement. The Lancet, 370, 1960-1973. 


\section{READING IN TIMES OF LOSS}

Tan, E. S. (1996). Emotion and the structure of narrative film. Film as an emotion machine. Mahwah (NJ): Erlbaum.

Wells, A., \& Hakanen, E. A. (1991). The emotional use of popular music by adolescents. Journalism Quarterly, 68, 445-454.

Zeelenberg, L. W., \& V. Spiertz. (1993). Depressie en bibliotherapie: Over het gebruik van boeken bij depressie. Doctoral dissertation. Utrecht University, Utrecht.

Zill, N., \& Winglee, M. (1988). Who reads literature? Survey data on the reading of fiction, poetry, and drama by U.S. adults during the 1980s. Washington, DC: National Endowment for the Arts.

Zisook, S. \& Kendler, K. S. (2007). Is bereavement-related depression different than nonbereavement-related depression? Psychological Medicine, 37, 779-794.

Zisook, S., \& Shuchter, S. R. (1991). Depression through the first year after the death of a spouse. The American Journal of Psychiatry, 148, 1346-1352. 